Research Article

\title{
Present Status and Future Perspectives for the EXO-200 Experiment
}

\author{
Giorgio Gratta ${ }^{1}$ and David Sinclair ${ }^{2,3}$ \\ ${ }^{1}$ Department of Physics, Stanford University, Stanford, CA, USA \\ ${ }^{2}$ Department of Physics, Carleton University, Ottawa, ON, Canada \\ ${ }^{3}$ TRIUMF, Vancouver, BC, Canada
}

Correspondence should be addressed to David Sinclair; sinclair@physics.carleton.ca

Received 22 July 2013; Accepted 10 October 2013

Academic Editor: Vincenzo Flaminio

Copyright (C) 2013 G. Gratta and D. Sinclair. This is an open access article distributed under the Creative Commons Attribution License, which permits unrestricted use, distribution, and reproduction in any medium, provided the original work is properly cited.

The EXO collaboration has built and operated a $200 \mathrm{~kg}$ liquid xenon detector for studies of double beta decay. This paper summarizes the results obtained so far and their significance. The excellent performance of the detector encourages the concept of a much larger detector to obtain improved sensitivity to the possible detection of the neutrinoless decay mode of xenon.

\section{Introduction}

The search for neutrinoless double beta decay is one of the most pressing but also one of the most challenging endeavours in the push to understand the natural world beyond the standard model of particle physics. The standard model has had great success in describing the interactions of the elementary particles and, with the recent observation of a Higgs-like particle, of explaining the mass of all particles except for the neutrinos. In 1937, Majorana [1] suggested that neutrinos might be described as 2-component Weyl spinors rather than the 4 component description of the Dirac model. Shortly after, Furry [2] pointed out that while the Majorana model would not change normal beta decay, it would allow a new form of double beta decay in which no neutrinos were emitted. More than 75 years later, we still have not detected this decay mode or resolved the fundamental difference in the Majorana and Dirac models for the neutrino.

The first evidence that rates for either mode of double beta decay that would be very long came from the observation of apparently stable isotopes in nature which were energetically able to decay by this mode. This sets a lower bound on the lifetimes comparable to geological timescales. The limits were extended to higher values by searches for possible daughter elements. For example, in the work of Inghram and Reynolds [3] tellurium bearing ores were analyzed for the presence of xenon isotopes. Limits on decay half-lives in the order of $10^{19}$ years were set. Direct searches for double beta decay using counters were also started at about this time. Searches for two electrons being emitted from ${ }^{124} \mathrm{Sn}$ were carried out using coincident electron counters by Fireman [4] and in a bubble chamber by Fireman and Schwarzer [5] gave a lower bound of $10^{17}$ years. A motivation for these early searches was the expectation that the neutrinoless decay would be much faster than the two-neutrino decay, if the neutrino was indeed a Majorana particle. This expectation changed dramatically when parity nonconservation in weak interactions was observed and the V-A behavior of the weak interaction established.

The first successful detection of the two-neutrino double beta decay mode was made by a group led by Moe (see Elliott et al. [6]). Electrons from a foil of ${ }^{82} \mathrm{Se}$ were detected in a TPC to give an unambiguous signature of the decay process. The observed half-life was about $10^{20}$ years. The twoneutrino decay mode of several metallic elements has now been measured with the most precise data coming from the NEMO [7] experiment.

Limits on neutrinoless double beta decay lifetimes continued to increase with the application of more sophisticated experimental techniques, but the recent observation of neutrino oscillations has greatly enhanced the interest in this 
search. Neutrino oscillations, as discovered for atmospheric neutrinos by Fukuda et al. [8], for solar neutrinos by Ahmad et al. [9] and for reactor neutrinos by Eguchi et al. [10] show that neutrinos must possess mass and that the individual lepton flavor numbers are not conserved quantities. These discoveries have been confirmed by a number of experiments that have, in many cases, improved on the precision of the parameters entering oscillation description. Some recent references can be found in [11-17].

Neutrino-antineutrino oscillations were first proposed by Pontecorvo [18] in analogy with the $K_{0}$ oscillation in the hadron sector. Pontecorvo and Gribov $[19,20]$ first suggested that the solar neutrino problem could be explained by flavor oscillation of neutrinos and this fundamental insight, with modifications due to matter effects, has now been demonstrated to be correct. The observation that neutrinos do oscillate is a challenge to the standard model because it cannot explain, without some extension, why particles always observed to be left handed can have mass.

Thus, the fundamental questions which can now be addressed by an observation of neutrinoless double beta decay are as follows.

(1) Are neutrinos Dirac or Majorana particles?

(2) Is total lepton number conserved or is it violated as required in some cosmological models?

(3) Can we determine the absolute mass scale for neutrinos?

There are several isotopes which can be exploited in the search for double beta decay. Germanium is attractive because of the advanced technology for making high resolution, high volume detectors. Tellurium is attractive because of the high natural abundance of the candidate isotope ${ }^{130} \mathrm{Te}$. In this paper, we describe a search for double beta decay in ${ }^{136} \mathrm{Xe}$.

\section{Xenon Detectors for Double Beta Decay}

There are several reasons why xenon is an attractive target for the double beta decay search. The most interesting isotope, ${ }^{136} \mathrm{Xe}$, is quite abundant at $9.6 \%$ in nature and it is one of the easiest (and hence least expensive) of the double beta decay candidates to enrich. It is possible to make xenon extremely pure and this is essential both to allow a detector to be built in which electrons drift without serious loss and to eliminate radioactive backgrounds which can mimic the double beta decay signal. There are no long-lived xenon isotopes which might give internal backgrounds. Xenon detectors may be in gaseous form (as used, e.g., in the Gotthard double beta decay search [21] and in the NEXT [22] projects) or as a liquid detector as used in EXO-200. Xenon may also be dissolved into a liquid scintillator as used at Gando et al. [23]. Each form has advantages and disadvantages. Gaseous detectors offer the possibility of excellent spatial information about the decays including a clear signature of two electron tracks. This has proven important in reducing gamma ray backgrounds [21]. The best gaseous detectors have offered near Fano limited energy resolutions [22], while this has not yet been reached with liquid detectors. The gas detectors can operate at room temperature and do not require complex cryogenics. Finally, gas detectors can operate with intrinsic gain and this greatly reduces the electronic noise contributions. Detectors which employ xenon dissolved into organic liquid scintillators offer excellent low background environments and a high degree of uniformity and homogeneity. The energy resolution has not yet matched that of other technologies and the detectors act as a pure calorimeter without any tracking information. On the other hand, condensed liquid xenon detectors are much more compact requiring much less underground space and shielding. Furthermore, the high density of liquid xenon allows very good self-shielding against external gamma backgrounds, moderate resolution, and the ability to reconstruct the event topologies. EXO-200 is a liquid phase detector.

\section{Barium Tagging}

A unique possibility offered by a xenon detector is that all backgrounds except the two-neutrino decay mode could be effectively removed by identification of the daughter barium ion. This possibility was first put forward by Moe [24] and is based on the observation by a number of groups of single ion detection for barium. Essentially, one transports the daughter ion to a region where it can be identified using atomic laser resonant spectroscopy. The EXO-200 detector does not include a barium tag provision, but the EXO group are actively pursuing technologies to accomplish this tag with both liquid and gas type detectors and this may prove essential for an ultimate double beta decay detector sensitive through the normal hierarchy of neutrino masses.

\section{The EXO-200 Detector}

The EXO-200 detector is in the form of a back-to-back liquidxenon time projection chambers (TPCs), with high efficiency UV light detection with a total inventory of $200 \mathrm{~kg}$ of xenon enriched to $81 \%$ in ${ }^{136} \mathrm{Xe}$. Both liquid and gas detectors require the scintillation signal to provide the start time signal, but, in addition, it was determined early in the development of the EXO detector that in liquid xenon the energy deposited by ionizing radiation is split between production of electrons and scintillation photons and that both must be measured to ensure a good energy resolution [25]. The detector has been described in detail in [26]. Figure 1 shows the details of the central detector element, while Figure 2 displays the overall detector arrangement.

4.1. Detector Operation. In an ionizing event, a burst of scintillation light at $175 \mathrm{~nm}$ is emitted. The intensity is approximately 50,000 photons per $\mathrm{MeV}$ for minimum ionizing particles. These photons are recorded using an array of large area avalanche photodiodes located behind the anode. All surfaces except the diodes are reflecting to maximize the detected yield.

Electrons liberated by the ionization drift towards the anode under the drift field of about $500 \mathrm{~V} / \mathrm{cm}$. At the anode, there is a pair of wire planes oriented 60 degrees to each 


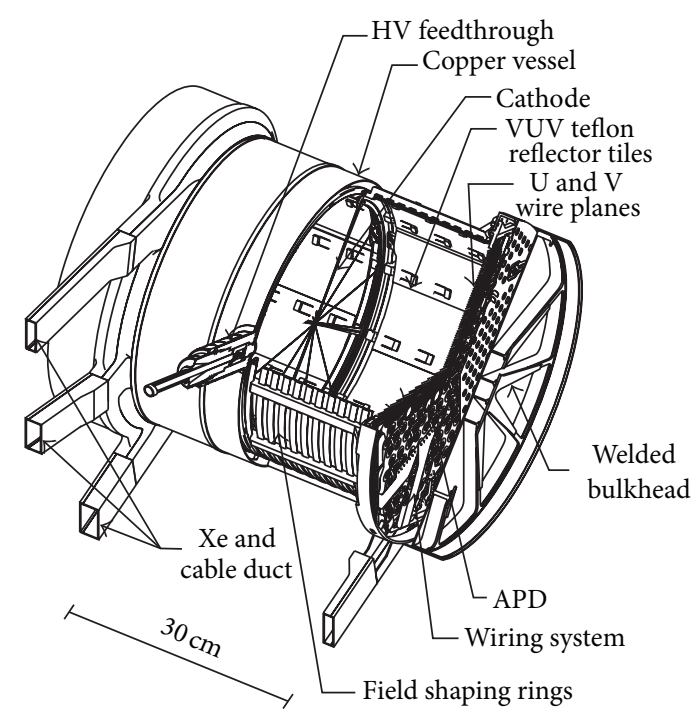

Figure 1: Details of the EXO-200 detector.

other. At the first plane, signals are induced by the passing electrons, while the electrons are collected on the second plane. An increased field between the wires gives high gridding efficiency. Signals from the wires are digitized at $1 \mathrm{MHz}$ so that the time evolution of the arriving electrons can be determined. From this information, it is possible to reconstruct the full 3-dimensional distribution of ionization. As discussed below, this information is critical for two aspects of the analysis - it allows for a definition of a fiducial boundary to maximize the signal to background ratio and it allows a separation between double beta-like events where the energy is deposited in a single location from the gamma-like events where the events tend to involve multiple Compton scatterings giving multisite ionization. The resolution is not adequate to see the electron tracks or to distinguish single from double electron events. Alpha-decay events can be readily identified by their very high light to ionization yield.

The detector is constructed as two back-to-back TPCs with a common central cathode. This configuration allows shorter average electron drift distances that, in turn, minimizes the electron loss due to attachment.

There are several aspects of the detector design that are aimed at controlling the radioactive background. All detector materials have been carefully screened for activity and a lengthy report on the assay program has been published [20]. The materials for the inner detector are mainly low activity copper, phosphor bronze, acrylic, and teflon. The central detector is surrounded by a heat transfer fluid HFE7000 [28] which provides a cooling link between the refrigeration systems and the detector and with a specific density of about 1.8 at liquid xenon temperatures provides a good gamma shield. The next layer is the copper cryostat formed from two layers of ultralow background copper of thickness $2.5 \mathrm{~cm}$. The cryostat is surrounded by $25 \mathrm{~cm}$ of low activity lead to provide the main shield against external gamma backgrounds. Finally, a set of cosmic ray veto detectors covers most of the surface to tag muon events. With this configuration, the residual backgrounds are mainly from the local detector materials. The detector is located in the WIPP facility in New Mexico, USA which provides an overburden of 1600 metres of water equivalent.

4.2. Detector Performance. The detector response is measured using a calibration facility that can move gamma sources to locations about the detector. Key parameters to be measured include the charge calibration for the wires, the light response as a function of position, the electron lifetime during drift, single site and multisite event ratios, and the overall energy scale.

The electron lifetime is an important parameter in establishing good energy resolution and it is a very sensitive measure of the xenon purity. In EXO-200, lifetimes of about $3 \mathrm{~ms}$ have been maintained. This is achieved by careful attention to cleanliness at all stages of the detector construction and a high recirculation flow through a SAES getter for impurity removal.

An important aspect of the analysis of EXO-200 data is the discrimination between gamma and beta events based on the number of ionization clusters. The discrimination depends on energy, but for events near the zero neutrino $Q$ value, the enhancement of gamma events in the multisite partition is about a factor of 5 (as seen in Figure 3). This is important not only for understanding the gamma background but also, if a peak were observed at $Q_{\beta \beta}$ in the single site spectrum, ruling for out a gamma interpretation if a much stronger peak were not observed in the corresponding multisite data.

The overall test of the calibration process is the extent to which the detector Monte Carlo can reproduce the observed response to known gamma sources. Such a test is displayed in Figure 3. The agreement is excellent.

\section{Experimental Results}

5.1. Two-Neutrino Double Beta Decay of ${ }^{136} \mathrm{Xe}$. The first low background physics run of EXO-200 produced the data displayed in Figure 4. Events are classified as single site or multisite, as was done for the calibration data. Now, most of the events involving gamma rays will appear as multisite because most gammas will Compton scatter in the detector. Most of the double beta events will show up at single sites because the range of the betas is very small in the liquid xenon compared with the spatial resolution. One can see that the data are completely dominated by the two-neutrino decay. That is, the backgrounds are typically less than $10 \%$ of the signal. One can also see that the fit of all known signals to the data gives an excellent description of the observed spectrum.

Prior to the EXO experiment no observation of the two-neutrino double beta decay mode had been reported although there were two reports of studies which produced only upper limits for the decay rate $[29,30]$. The reported bounds were of concern because they suggested that the two-neutrino decay of ${ }^{136} \mathrm{Xe}$ was anomalously low. The EXO collaboration first reported a clear observation of the two neutrino decay mode in 2011 [31]. The rate seen was considerably higher than the limits previously reported and reflects 


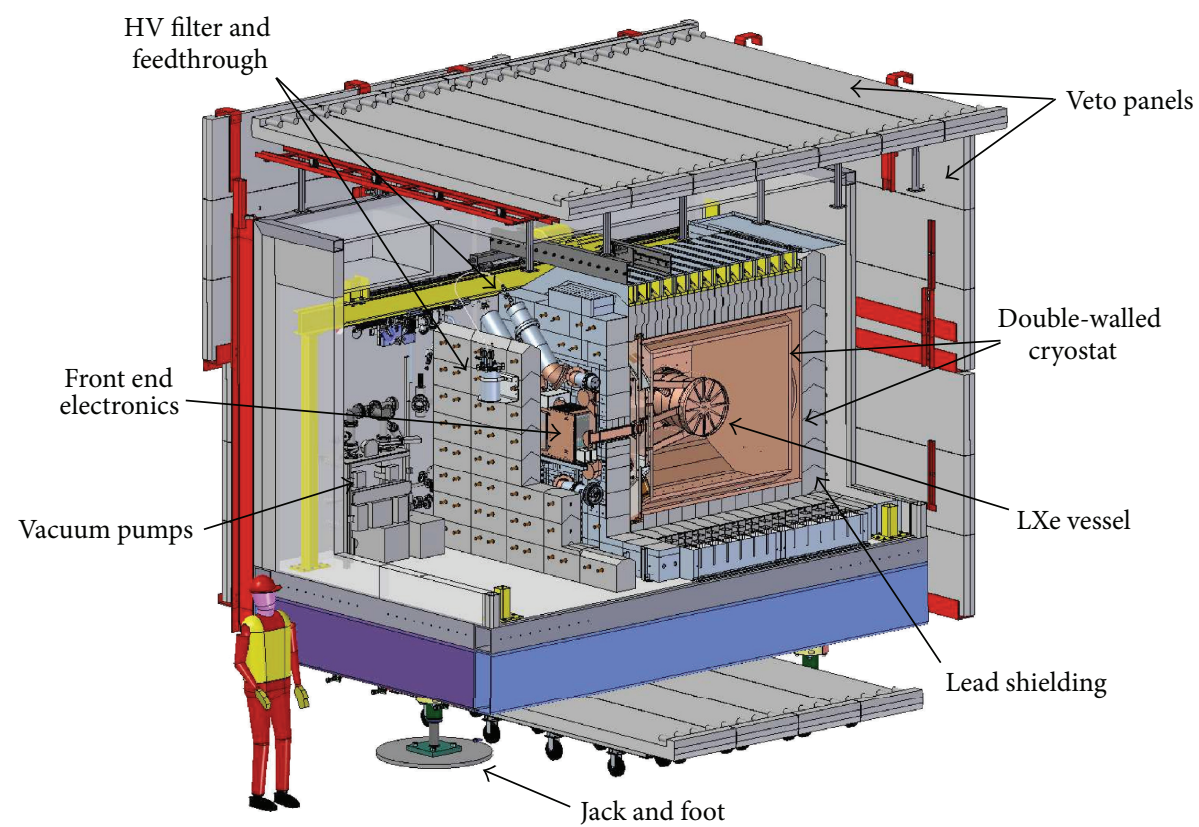

FIgURE 2: Overall detector configuration as installed at WIPP.
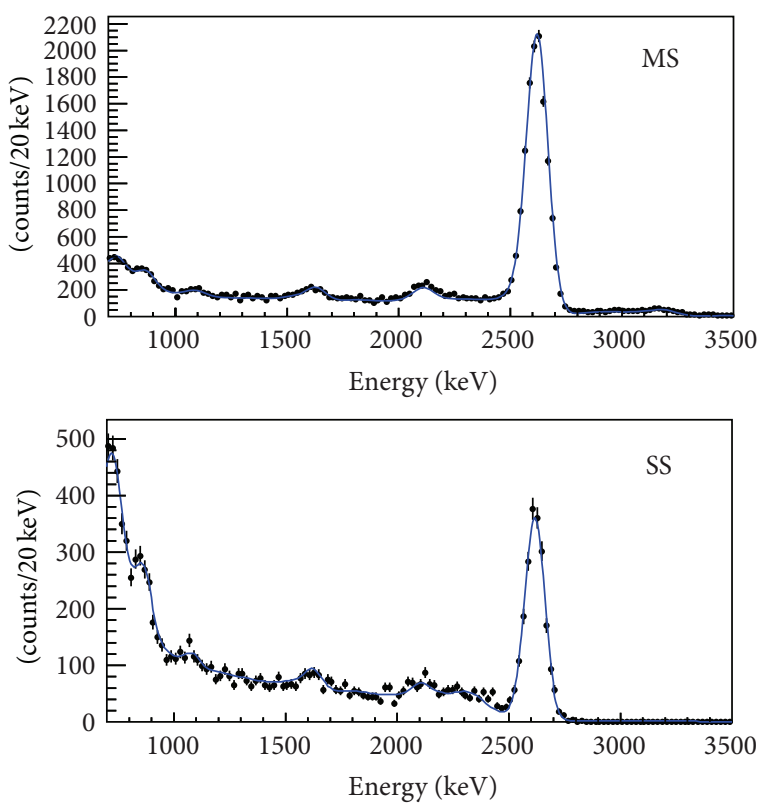

Figure 3: Multisite and single site data and Monte Carlo for a ${ }^{228} \mathrm{Th}$ source [25]. Excellent quantitative agreement is observed demonstrating that the detector is well characterized.

the advantages of a large mass, very low background detector. The EXO results were confirmed by the KAMLAND-ZEN team shortly after [24]. In a paper recently submitted for publication, the EXO group have presented a fuller analysis where several of the systematic errors have been reduced to provide the highest precision measure of a two-neutrino double beta decay. The final result is a half-life of $2.172 \pm 0.017$ (stat) $\pm 0.060($ sys $) \times 10^{21}$ years [32].
5.2. Search for Zero Neutrino Decays of ${ }^{136} \mathrm{Xe}$. The primary aim of the EXO project is the search for neutrino-less decay of ${ }^{136} \mathrm{Xe}$. The signature of such a decay would be a peak in the total deposited energy spectrum at the decay $Q$ value. The EXO-200 first search for this decay mode was published in 2012 [27] where details of the analysis may be found. The data are displayed in Figure 4. Critical to the search for the neutrino-less decay is the separation between gamma backgrounds and the signal through the multisite/single site discrimination. No evidence for neutrino-less decay is observed.

As discussed in [27], the analysis of these data gives a bound on the possible neutrinoless double beta decay of ${ }^{136} \mathrm{Xe} T>1.6 \times 10^{25}$ years at $90 \%$ confidence. To extract a corresponding limit to the neutrino mass, one needs to choose a nuclear matrix element. For recently published values using generator coordinate method [33], nuclear shell model [34], interacting boson model [35], RQRPA [36], and the QRPA-2 [37], the mass limit is $140-380 \mathrm{meV}$.

There has been a claim of observation of neutrinoless double beta decay in germanium [38]. In order to compare the EXO limits to expectations for germanium, one uses the relative matrix elements from a set of calculations. The results are displayed in Figure 5. Clearly the measurement from EXO does not support the previous claim. Again, the EXO result has been confirmed by Gando et al. [39].

\section{General Discussion of Strengths of the Liquid Xenon Detection and Future Directions}

The EXO-200 experiment has provided world-leading measures of two-neutrino double beta decay and limits for neutrino-less decay of ${ }^{136} \mathrm{Xe}$. There are a number of reasons 

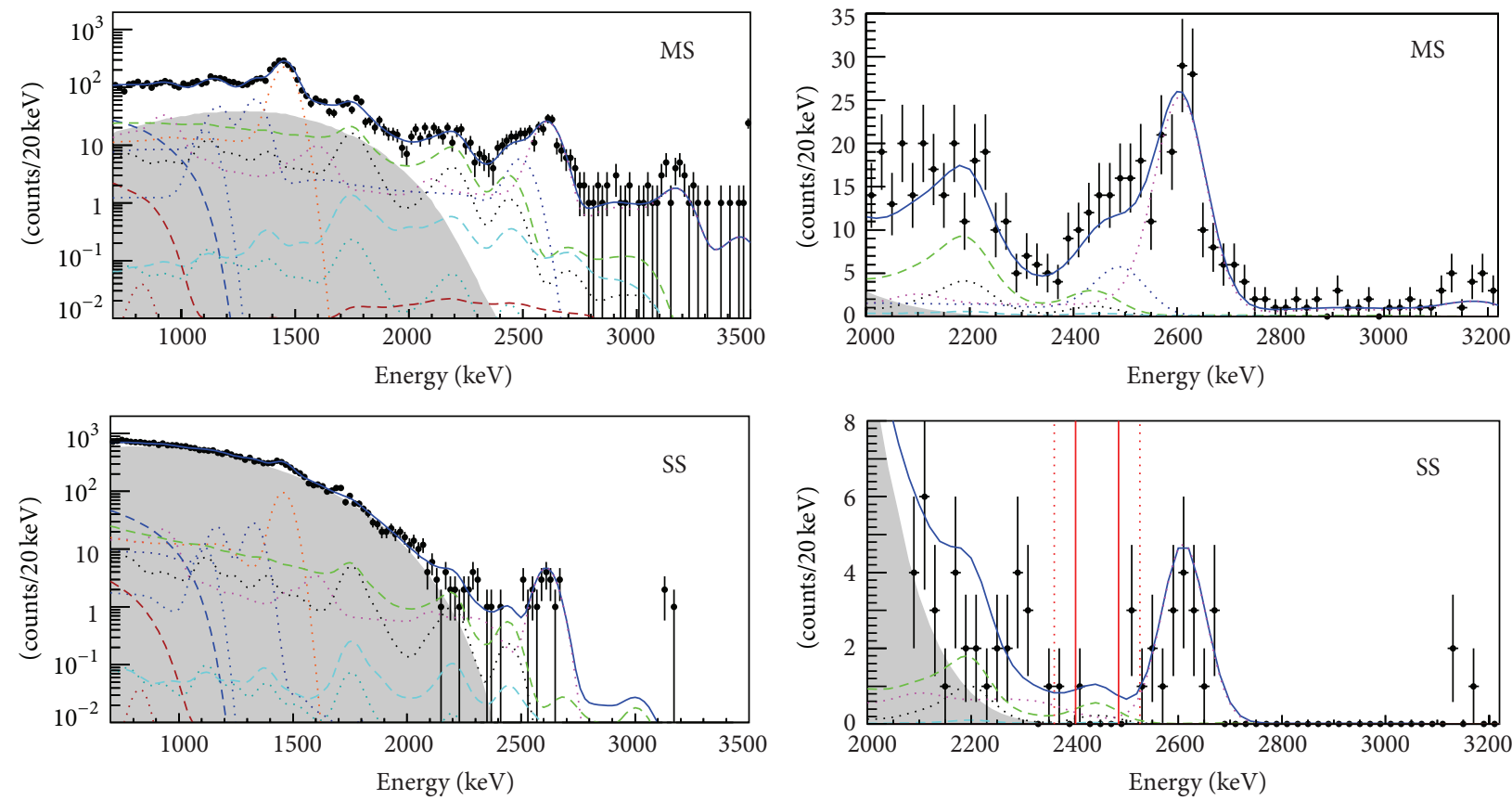

FIgURE 4: The EXO data [27] showing the multisite events and the single site events. The best fit is shown in blue, while the background components are as follows: grey: $2 \nu \beta \beta$; dotted orange: ${ }^{40} \mathrm{~K}$; dark blue: ${ }^{60} \mathrm{Co}$; green, cyan and black are ${ }^{238} \mathrm{U}$ (mainly ${ }^{214} \mathrm{Bi}$ ) on the air gap, TPC vessel, and cathode, respectively; dotted magenta: ${ }^{232} \mathrm{Th}$ in the vessel.

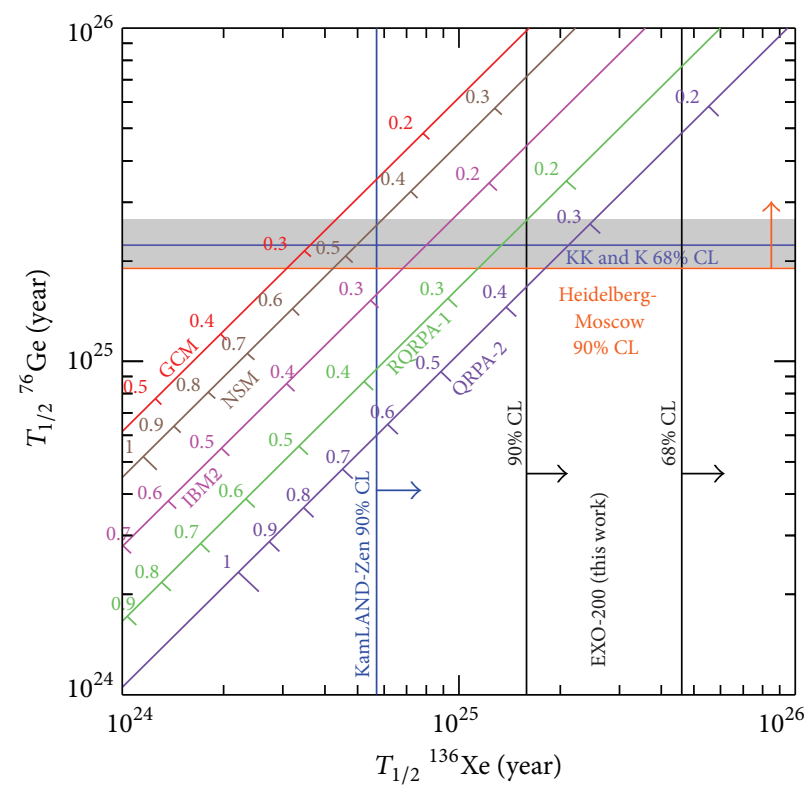

FiguRE 5: This figure compares the expected half-lives of ${ }^{136} \mathrm{Xe}$ and ${ }^{76} \mathrm{Ge}$ for matrix elements from various models. References for the models are found in the text.

for this success. Clearly, a large mass detector is essential for a low decay rate study, but the EXO design offers a unique suite of advantages. The precision measure is in part due to the highly homogeneous nature of the detector. This allows very tight control on the systematic errors. The rejection of the gamma background arises first from the choice of very clean materials, second from the ability to determine the full structure of the events and thus discriminate against gamma rays that tend to Compton scatter, and finally from the ability to set a fiducial boundary inside the xenon mass. The background is qualitatively different from that found for germanium or tellurium experiments in that, as can be seen from Figure 5, there is very little continuum beneath the peak region. Instead, we need to reject two discrete gamma activities $\left(2448 \mathrm{keV}\right.$ from ${ }^{214} \mathrm{Bi}$ and the $2505 \mathrm{keV}$ sum peak from ${ }^{60} \mathrm{Co}$ ) with energies very close to $Q_{\beta \beta}$ together with 
the tail of the $2614 \mathrm{keV}$ gamma line from thorium. Improved rejection of all of these backgrounds will likely come from better single site/multisite discrimination, whereas better energy resolution will benefit mainly the 2614 rejection. Significant improvements to the multi-site identification look possible.

\section{The Future}

Many of the detector concepts and implementation in EXO200 are readily scalable to a much larger sensitive mass of xenon. Indeed, the critical self-shielding improves with larger mass. With the extremely high purity level demonstrated for xenon, it should be possible to cover the inverted hierarchy in a sufficiently large detector. The EXO collaboration is planning a new, next generation detector using 5 tonnes of enriched xenon. The detector would be placed in a large water shield instead of the lead shield used for EXO-200 and a deeper site would be chosen to reduce the cosmogenic neutron backgrounds. The plan is to run initially without a barium tag but to design the facility so that such a tag could be implemented at a later time. Many physics and engineering studies are underway to explore the full capability of such a detector, but it is clear that such a detector would allow a very substantial advance in the sensitivity to a Majorana neutrino mass and, with a barium tag, it would be possible to cover the entire inverted hierarchy parameter space. This is clearly the next milestone in this exciting area of research. Much work remains to be done, but it seems reasonable to think that this exciting project could be ready to start data taking a decade from now.

\section{References}

[1] E. Majorana, "Teoria simmetrica dell'elettrone e del positrone," Il Nuovo Cimento, vol. 14, no. 4, pp. 171-184, 1937.

[2] W. H. Furry, "Note on the theory of the neutral particle," Physical Review, vol. 54, no. 1, pp. 56-67, 1938.

[3] M. G. Inghram and J. H. Reynolds, "On the double beta-process," Physical Review, vol. 76, no. 8, pp. 1265-1266, 1949.

[4] E. L. Fireman, "A measurement of the half-life of double betadecay from ${ }_{50} \mathrm{Sn}^{124}$," Physical Review, vol. 75, no. 2, pp. 323-324, 1949.

[5] E. L. Fireman and D. Schwarzer, "A re-investigation of the double beta-decay from $\mathrm{Sn}^{124}$," Physical Review, vol. 86, no. 4, pp. 451-453, 1952.

[6] S. R. Elliott, A. A. Hahn, and M. K. Moe, "Direct evidence for two-neutrino double-beta decay in ${ }^{82}$ Se," Physical Review Letters, vol. 59, no. 18, pp. 2020-2023, 1987.

[7] NEMO, http://nemo.in2p3.fr/nemow3/publications.html.

[8] Y. Fukuda, T. Hayakawa, E. Ichihara et al., "Evidence for oscillation of atmospheric neutrinos," Physical Review Letters, vol. 81, no. 8, pp. 1562-1567, 1998.

[9] Q. R. Ahmad, R. C. Allen, T. C. Andersen et al., "Direct evidence for neutrino flavor transformation from neutral-current interactions in the sudbury neutrino observatory," Physical Review Letters, vol. 89, no. 1, Article ID 011301, 6 pages, 2002.

[10] K. Eguchi, S. Enomoto, K. Furuno et al., "First results from KamLAND: evidence for reactor antineutrino disappearance,"
Physical Review Letters, vol. 90, no. 2, Article ID 021802, 6 pages, 2003.

[11] P. Adamson, I. Anghel, C. Backhouse et al., "Measurement of neutrino and antineutrino oscillations using beam and atmospheric data in MINOS," Physical Review Letters, vol. 110, no. 25, Article ID 251801, 6 pages, 2013.

[12] K. Abe, N. Abgrall, H. Aihara et al., "Measurement of neutrino oscillation parameters from muon neutrino disappearance with an off-axis beam," http://arxiv.org/abs/1308.0465.

[13] N. Agafonova, A. Aleksandrov, A. Anokhina et al., "New results on $v_{\mu} \rightarrow v_{\tau}$ appearance with the OPERA experiment in the CNGS beam," http://arxiv.org/abs/1308.2553.

[14] M. H. Ahn, E. Aliu, S. Andringa et al., "Measurement of neutrino oscillation by the K2K experiment," Physical Review D, vol. 74, no. 7, Article ID 072003, 39 pages, 2006.

[15] F. P. An, Q. An, J. Z. Bai et al., "Improved measurement of electron antineutrino disappearance at Daya Bay," Chinese Physics C, vol. 37, no. 1, 2013.

[16] J. K. Ahn, S. Chebotaryov, J. H. Choi et al., "Observation of reactor electron antineutrinos disappearance in the RENO experiment," Physical Review Letters, vol. 108, no. 19, Article ID 191802, 6 pages, 2012.

[17] M. S. Berger, "Higgs sector radiative corrections and s-channel production," Physical Review Letters, vol. 87, no. 13, Article ID 131801, 4 pages, 2001.

[18] B. Pontecorvo, J. Exp. Theor. Phys., vol. 33, p. 549, 1957.

[19] B. Pontecorvo, "Neutrino experiments and the problem of conservation of leptonic charge," Zhurnal Eksperimental'noi $i$ Teoreticheskoi Fiziki, vol. 53, pp. 1717-1725, 1967.

[20] V. Gribov and B. Pontecorvo, "Neutrino astronomy and lepton charge," Physics Letters B, vol. 28, no. 7, pp. 493-496, 1969.

[21] R. Luescher, J. Farine, F. Boehm et al., "Search for $\beta \beta$ decay in ${ }^{136}$ Xe: new results from the Gotthard experiment," Physics Letters B, vol. 434, no. 3-4, pp. 407-414, 1998.

[22] V. Álvarez, F. I. G. Borges, S. Cárcel et al., "Ionization and scintillation response of high-pressure xenon gas to alpha particles," Journal of Instrumentation, vol. 8, Article ID P05025, 2013.

[23] A. Gando, Y. Gando, and H. Hanakago, "Measurement of the double- $\beta$ decay half-life of ${ }^{136} \mathrm{Xe}$ with the KamLAND-Zen experiment," Physical Review C, vol. 85, no. 4, Article ID 045504, 6 pages, 2012.

[24] M.K. Moe, "Detection of neutrinoless double-beta decay," Physical Review C, vol. 44, no. 3, pp. R931-R934, 1991.

[25] E. Conti, R. DeVoe, G. Gratta et al., "Correlated fluctuations between luminescence and ionization in liquid xenon," Physical Review B, vol. 68, no. 5, Article ID 054201, 5 pages, 2003.

[26] M. Auger, D. J. Auty, P. S. Barbeau et al., "The EXO-200 detector, part I: detector design and construction," Journal of Instrumentation, vol. 7, Article ID P05010, 2012.

[27] M. Auger, D. J. Auty, P. S. Barbeau et al., "Search for neutrinoless double-beta decay in ${ }^{136}$ Xe with EXO-200," Physical Review Letters, vol. 109, no. 3, Article ID 032505, 6 pages, 2012.

[28] C.-J. Kuo and Y. Peles, "Flow boiling of coolant (HFE-7000) inside structured and plain wall microchannel," Journal of Heat Transfer, vol. 131, no. 12, pp. 1-9, 2009.

[29] R. Bernabei, P. Belli, F. Cappella et al., "Investigation of $\beta \beta$ decay modes in ${ }^{134} \mathrm{Xe}$ and ${ }^{136} \mathrm{Xe}$," Physics Letters B, vol. 546, no. 1-2, pp. 23-28, 2002. 
[30] J. M. Gavriljuk, A. M. Gangapshev, V. V. Kuzminov, S. I. Panasenko, and S. S. Ratkevich, "Results of a search for $2 \beta$ decay of ${ }^{136} \mathrm{Xe}$ with high-pressure copper proportional counters in Baksan neutrino observatory," Physics of Atomic Nuclei, vol. 69, no. 12, pp. 2129-2133, 2006.

[31] N. Ackerman, B. Aharmim, M. Auger et al., "Observation of two-neutrino double-beta decay in ${ }^{136} \mathrm{Xe}$ with the EXO-200 detector," Physical Review Letters, vol. 107, no. 21, Article ID 212501, 5 pages, 2011.

[32] J.B. Albert, M. Auger, and D.J. Auty, "An improved measurement of the $2 \nu \beta \beta$ half-life of ${ }^{136} \mathrm{Xe}$ with EXO-200," Physical Review. Submitted http://arxiv.org/abs/1306.6106.

[33] T. R. Rodríguez and G. Martínez-Pinedo, "Energy density functional study of nuclear matrix elements for neutrinoless $\beta \beta$ decay," Physical Review Letters, vol. 105, Article ID 252503, 4 pages, 2010.

[34] J. Menéndeza, A. Povesa, E. Caurierb, and F. Nowackib, "Disassembling the nuclear matrix elements of the neutrinoless $\beta \beta$ decay," Nuclear Physics A, vol. 818, no. 3-4, pp. 139-151, 2009.

[35] J. Barea and F. Iachello, "Neutrinoless double- $\beta$ decay in the microscopic interacting boson model," Physical Review C, vol. 79, no. 4, Article ID 044301, 16 pages, 2009.

[36] F. Šimkovic, A. Faessler, H. Müther, V. Rodin, and M. Stauf, " $0 \nu \beta \beta$-decay nuclear matrix elements with self-consistent short-range correlations," Physical Review C, vol. 79, no. 5, Article ID 055501, 10 pages, 2009.

[37] A. Staudt, K. Muto, and H. V. Klapdor-Kleingrothaus, "Calculation of $2 v$ and $0 v$ double-beta decay rates," Europhysics Letters, vol. 13, no. 1, 1990.

[38] H. V. Klapdor-Kleingrothaus and I. V. Krivosheina, “The evidence for the observation of $0 \nu \beta \beta$ decay: the identification of $0 \nu \beta \beta$ events from the full spectra," Modern Physics Letters A, vol. 21, no. 20, pp. 1547-1566, 2006.

[39] A. Gando, Y. Gando, H. Hanakago et al., "Limit on neutrinoless $\beta \beta$ decay of ${ }^{136} \mathrm{Xe}$ from the first phase of KamLAND-Zen and comparison with the positive claim in ${ }^{76} \mathrm{Ge}$," Physical Review Letters, vol. 110, no. 6, Article ID 062502, 5 pages, 2013. 

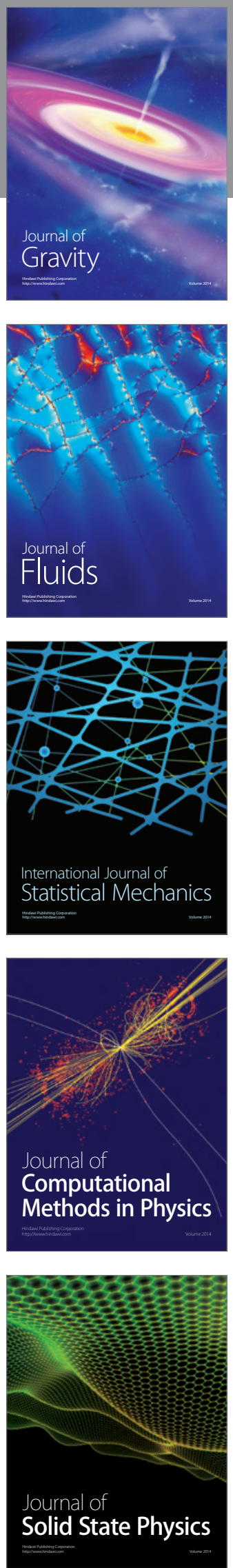

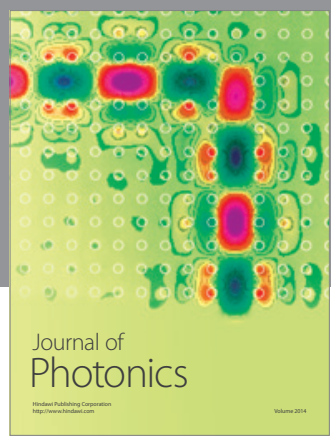

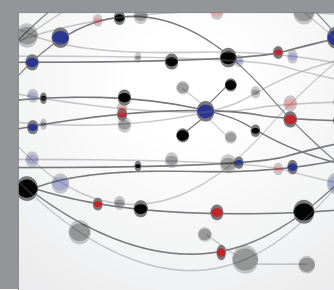

The Scientific World Journal

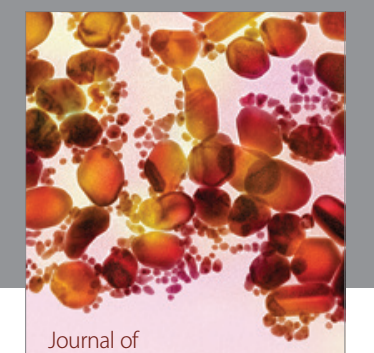

Soft Matter
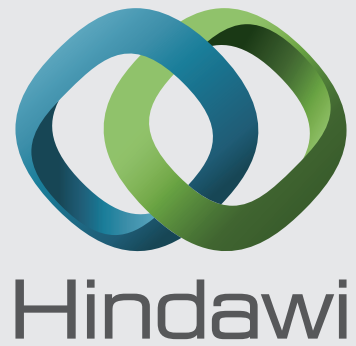

Submit your manuscripts at

http://www.hindawi.com
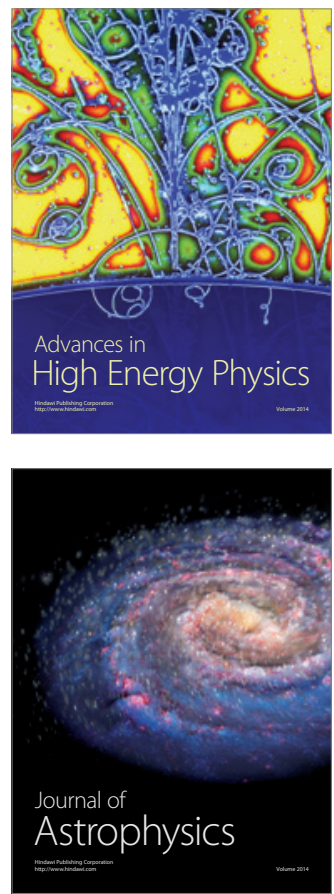
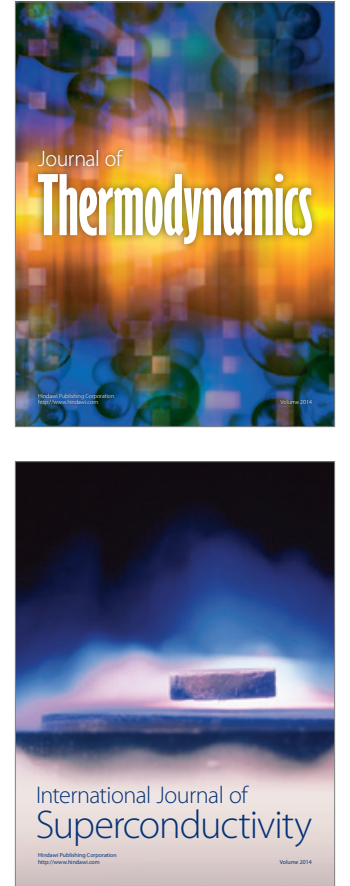
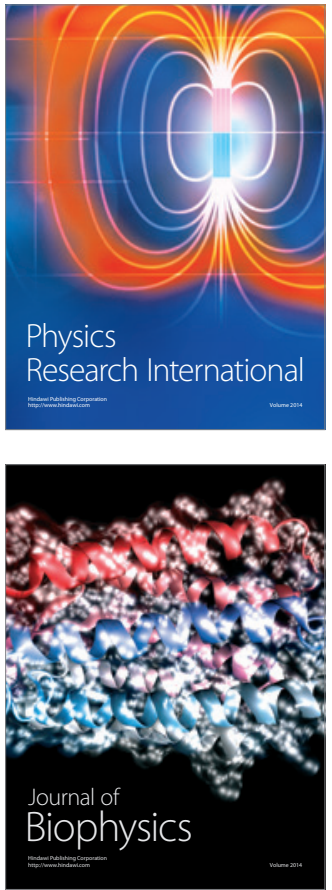
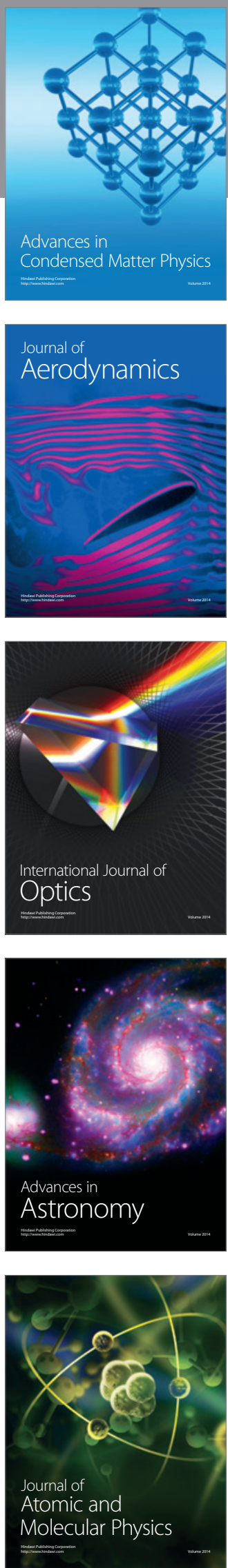